\title{
Synthetic Weyl Points and Chiral Anomaly in Majorana Devices with Nonstandard Andreev-Bound-State Spectra
}

Kotetes, Panagiotis; Mercaldo, Maria Teresa; Cuoco, Mario

\section{Published in:}

Physical Review Letters

DOI:

10.1103/PhysRevLett.123.126802

Publication date:

2019

\section{Document version}

Publisher's PDF, also known as Version of record

Citation for published version (APA):

Kotetes, P., Mercaldo, M. T., \& Cuoco, M. (2019). Synthetic Weyl Points and Chiral Anomaly in Majorana Devices with Nonstandard Andreev-Bound-State Spectra. Physical Review Letters, 123(12), [126802]. https://doi.org/10.1103/PhysRevLett.123.126802 


\title{
Synthetic Weyl Points and Chiral Anomaly in Majorana Devices with Nonstandard Andreev-Bound-State Spectra
}

\author{
Panagiotis Kotetes $\odot,{ }^{1,2, *}$ Maria Teresa Mercaldo, ${ }^{3}$ and Mario Cuoco ${ }^{4,3}$ \\ ${ }^{1}$ CAS Key Laboratory of Theoretical Physics, Institute of Theoretical Physics, Chinese Academy of Sciences, \\ Beijing 100190, China \\ ${ }^{2}$ Niels Bohr Institute, University of Copenhagen, 2100 Copenhagen, Denmark \\ ${ }^{3}$ Dipartimento di Fisica “E. R. Caianiello," Università di Salerno, IT-84084 Fisciano (SA), Italy \\ ${ }^{4}$ CNR-SPIN, IT-84084 Fisciano (SA), Italy
}

(Received 12 January 2019; revised manuscript received 10 August 2019; published 19 September 2019)

\begin{abstract}
We demonstrate how to design various nonstandard types of Andreev-bound-state (ABS) dispersions, via a composite construction relying on Majorana bound states (MBSs). Here, the MBSs appear at the interface of a Josephson junction consisting of two topological superconductors (TSCs). Each TSC harbors multiple MBSs per edge by virtue of a chiral or unitary symmetry. We find that, while the ABS dispersions are $2 \pi$ periodic, they still contain multiple crossings which are protected by the conservation of fermion parity. A single junction with four interface MBSs and all MBS couplings fully controllable, or networks of such coupled junctions with partial coupling tunability, open the door for topological band structures with Weyl points or nodes in synthetic dimensions, which in turn allow for fermion-parity (FP) pumping with a cycle set by the ABS-dispersion details. In fact, in the case of nodes, the FP pumping is a manifestation of chiral anomaly in 2D synthetic spacetime. The possible experimental demonstration of ABS engineering in these devices further promises to unveil new paths for the detection of MBSs and higher-dimensional chiral anomaly.
\end{abstract}

DOI: 10.1103/PhysRevLett.123.126802

Superconducting devices are currently in the spotlight [1], with Josephson junctions of conventional superconductors (SCs) being the core for state-of-the-art quantum-computing circuits $[2,3]$. Each junction is characterized by the superconducting phase difference $\Delta \phi$ across it and the associated Josephson energy scale $E_{J}(\Delta \phi)[4,5]$. When the Josephson link is a quantum-point contact or a quantum dot, the resulting transport [6-9] is mainly mediated by a small number of electronic so-called Andreev bound states (ABSs) [10], which are localized near the interface. For a junction of two conventional SCs, the ABS energy dispersions come in pairs [7-9], $\varepsilon(\Delta \phi)= \pm E_{\mathrm{ABS}} \sqrt{1-\mathcal{T} \sin ^{2}(\Delta \phi / 2)}$, with $\mathcal{T}$ denoting the transparency of the junction and $E_{\mathrm{ABS}}$ an energy scale usually coinciding with the bulk gap of the SCs [11]. For a perfectly transparent junction, the ABS dispersions exhibit a linear crossing at $\Delta \phi=\pi[6]$. This is not protected, and a gap opens for $\mathcal{T} \neq 1$. In the high-transparency and lowinductance regime of the circuit, a pair of ABS levels can be employed to define a qubit [11], with $\Delta \phi=\pi$ being the operational sweet-spot value.

Perspectives for ABS engineering and novel quantumcomputing architectures open up in junctions of topological superconductors (TSCs). These may be either intrinsic, e.g., $p$-wave SCs [12-27], or artificial [28-37], arising in hybrid devices [38-58]. Both types harbor zero-energy and charge-neutral Majorana bound states (MBSs) [14,15,17]. Remarkably, a Josephson junction of two TSCs features pairs of coupled MBSs which give rise to $4 \pi$-periodic ABS energy dispersions [17,59-67], i.e., $\propto \cos (\Delta \phi / 2)$. In contrast to the conventional $s$ wave and unconventional $d$ wave $[68,69]$ ABS cases, here, the linear crossing at $\Delta \phi=\pi$ is protected by the conservation of fermion parity (FP) $[60,62]$, i.e., the number of electrons in the system modulo 2. The exotic properties of the MBSs, and their promise for fault-tolerant quantum computing [15,70-73], recently sparked their intense pursuit and resulted in their spectroscopic detection in various artificial platforms [74-92]. The next milestone is the demonstration of quantum manipulations [93-95] using MBSs. However, this is currently a challenging feat and, hence, it is urgent to unveil new phenomena that take advantage of the topological character of MBSs.

In this Letter, we propose to employ MBSs as building blocks for engineering various nonstandard ABS energy dispersions, which further unlock new types of Berry-phase $[96,97]$ and chiral-anomaly effects [98]. For this purpose, we consider junctions consisting of two TSCs, with each one harboring multiple zero-energy MBSs per edge by virtue of unitary or chiral symmetries [34,99-104]. We consider the minimal construction, where each TSC contributes with two MBSs per edge, i.e., $\gamma_{1,2}$ and $\gamma_{3,4}$. The resulting four MBSs can be pairwise coupled by terms $\left(t_{12,34}\right)$ violating the symmetries protecting the multiple MBSs before the TSCs get in contact, as well as by Majorana-Josephson terms $\left(t_{13,24,14,23}\right)$ originating from electron tunneling across the junction. The latter show a 
$4 \pi$-periodic dependence in terms of the phase difference [17,59-67], i.e., $t_{n m} \propto \cos \left[\left(\Delta \phi+\beta_{n m}\right) / 2\right]$, with $n m=13$, $24,14,23$. The phases $\beta_{n m}$ are determined by the symmetries dictating the two TSCs and the interface.

We mainly find $2 \pi$-periodic ABS dispersions which, for $t_{14,23}=0$, follow the form $\varepsilon(\Delta \phi) \propto \eta \cos \chi+\cos (\Delta \phi+\varphi)$. This situation takes place when the MBS state vectors differ across the junction only by phase factors set by $\varphi$ and $\chi$, and the interface preserves the symmetries of the two TSCs. The parameter $\eta$ controls the relative strength of the intraand inter-TSC MBS couplings. Apart from fully gapped spectra, we also obtain novel types of gapless dispersions, featuring either multiple FP-protected linear crossings or quadratic band touchings.

We show that such ABS spectra can be harnessed to engineer novel types of topologically nontrivial gapped band structures in synthetic spaces (cf. Refs. [105-108]) containing Berry monopoles [96,97], i.e., points in parameter space where band touchings occur. These become already accessible in 1D TSC junctions with a full gap. Alternatively, one can employ networks of 1D TSC junctions with gapless spectra to obtain fully gapped nontrivial bands. The presence of Berry monopoles allows for Cooper pair charge pumping $[109,110]$ in the manner of Thouless [111], by simultaneously sweeping $\Delta \phi$ and an additional parameter $\theta$. A full cycle pumps an integer number of $2 e$ charge which, however, does not switch the FP. Hence, to enable $1 e$ charge transfer and thus FP pumping $[102,112]$, we propose to sweep in $(\Delta \phi, \theta)$ space selectively. Thus, the fractional contribution of each Berry monopole can be isolated. Notably, the aspect of selective sweeping and its applicability to TSCs with multiple MBS which are not necessarily Kramers pairs differentiates our $\mathbb{Z}$-FP pump from the $\mathbb{Z}_{2}$-FP pump of Ref. [112].

We now present our general approach to the composite construction of the ABSs, by employing a minimum of four MBSs which appear near the junction's interface. The corresponding MBS operators satisfy $\left\{\gamma_{n}, \gamma_{m}^{\dagger}\right\}=\delta_{n m}$, with $n, m=1,2,3,4$. Since the MBSs define zero-energy quasiparticle excitations, $\gamma_{n}=\gamma_{n}^{\dagger}$ for all $n=1,2,3,4$, which further leads to the nonstandard relations $\gamma_{n}^{2}=1 / 2$. Each pair of MBSs appearing on the edge of each TSC is protected by chiral or unitary symmetries. The low-energy Hamiltonian obtained by projecting onto the MBS subspace reads $\mathcal{H}=\sum_{n, m=1,2,3,4}^{n<m} i \gamma_{n} t_{n m} \gamma_{m}$, with the coupling matrix elements $t_{12}$ and $t_{34}\left(t_{13,24,14,23}\right)$ having an intra-TSC (inter-TSC) character. These, generally depend on the superconducting phase difference biasing the junction. Here, we restrict to ABS spectra twisted by a charge-phase difference $\Delta \phi$. As we thoroughly discuss in our companion paper, Ref. [113], intrinsic spin-triplet $p$-wave SCs allow for the possibility of separately imposing a phase difference $\Delta \phi_{\uparrow, \downarrow}$ in each spin sector, which can be split into chargeand spin-phase components.
We reexpress the MBS Hamiltonian as follows:

$\mathcal{H}=\frac{i}{2} \boldsymbol{\Gamma}^{\mathrm{T}} \hat{B} \boldsymbol{\Gamma}=\frac{1}{2} \sum_{s= \pm}\left(\begin{array}{ll}a_{s}^{\dagger} & a_{s}\end{array}\right)\left(\begin{array}{cc}\varepsilon_{s} & 0 \\ 0 & -\varepsilon_{s}\end{array}\right)\left(\begin{array}{c}a_{s} \\ a_{s}^{\dagger}\end{array}\right)$,

where we employed the Majorana multicomponent operator $\boldsymbol{\Gamma}^{\boldsymbol{\top}}=\left(\gamma_{1} \gamma_{2} \gamma_{4} \gamma_{3}\right)$, its transpose $\boldsymbol{\Gamma}$, and the skewsymmetric matrix $\hat{B}$. Moreover, we introduced the ABS fermionic operators $a_{ \pm}$, satisfying $\left\{a_{s}, a_{p}^{\dagger}\right\}=\delta_{s, p}$ and $\left\{a_{s}, a_{p}\right\}=0$, with $s, p= \pm$. The ABS dispersions read:

$\varepsilon_{+}=\sqrt{\mathcal{S}+\sqrt{\mathcal{S}^{2}-[\operatorname{Pf}(\hat{B})]^{2}}}$ and $\varepsilon_{+} \varepsilon_{-}=\operatorname{Pf}(\hat{B})$,

with $\mathcal{S}=\sum_{n, m=1,2,3,4}^{n<m} t_{n m}^{2} / 2$ and the Pfaffian $\operatorname{Pf}(\hat{B})=$ $t_{13} t_{24}-t_{12} t_{34}-t_{14} t_{23}$. An equivalent expression was found in Ref. [65], which focused on different aspects of multiple MBSs than the ones considered here. We insist to express $\varepsilon_{-}$as in Eq. (2), obtained by $\left(\varepsilon_{+} \varepsilon_{-}\right)^{2}=[\operatorname{Pf}(\hat{B})]^{2}$, since this form reflects the antisymmetry of the MBS couplings [17], and further allows for a transparent description of ABS dispersions with protected crossings.

We move on with inferring the $\mathrm{ABS}$ dispersions obtained for the three limiting scenarios of Fig. 1, in which $t_{14,23}=0, t_{13} \propto t_{13}^{(0)} \cos [(\Delta \phi+\varphi+\chi) / 2]$, and $t_{24} \propto$ $t_{24}^{(0)} \cos [(\Delta \phi+\varphi-\chi) / 2]$, with $t_{n m}^{(0)}$ the MBS couplings obtained for $\Delta \phi=0$. Here, the interface preserves the symmetries of the two TSCs before contact, which further implies that the intra-TSC coupling elements $t_{12,34}$ are independent of $\Delta \phi$, i.e., $t_{12}=t_{12}^{(0)}$ and $t_{34}=t_{34}^{(0)}$.

$\left|t_{13}^{(0)}\right|,\left|t_{24}^{(0)}\right| \ll\left|t_{12}^{(0)}\right|,\left|t_{34}^{(0)}\right|$.- - In this case, the inter-TSC MBS couplings are considered to be substantially weaker than the intra-TSC ones, a situation which is feasible by reducing the junction's transparency. At the same time, $\left|t_{12}\right|$ and $\left|t_{34}\right|$ are still considered much smaller than the bulk energy gap, so that the present low-energy projection remains valid. Given the above, we find that both pairs
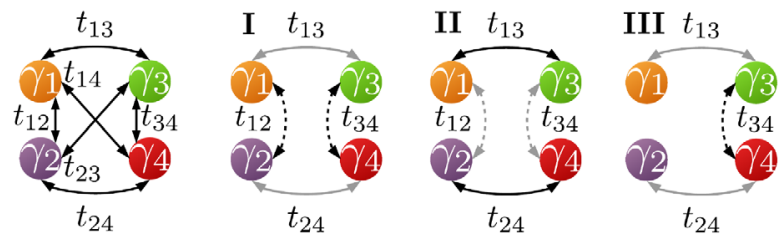

FIG. 1. Majorana-bound-state (MBS) couplings for four MBSs appearing at the interface of a junction formed by two topological superconductors (TSCs). Each TSC provides a pair of decoupled or hybridized MBSs, before contact. The couplings are divided into intra-TSC $t_{12,34}$ and inter-TSC $t_{13,24,14,23}$. We focus on three representative scenarios I, II, and III, depending on the strengths of the couplings. The dark and light colored arrows denote the strong and weak MBS couplings. If a line is missing, the respective coupling is zero. 

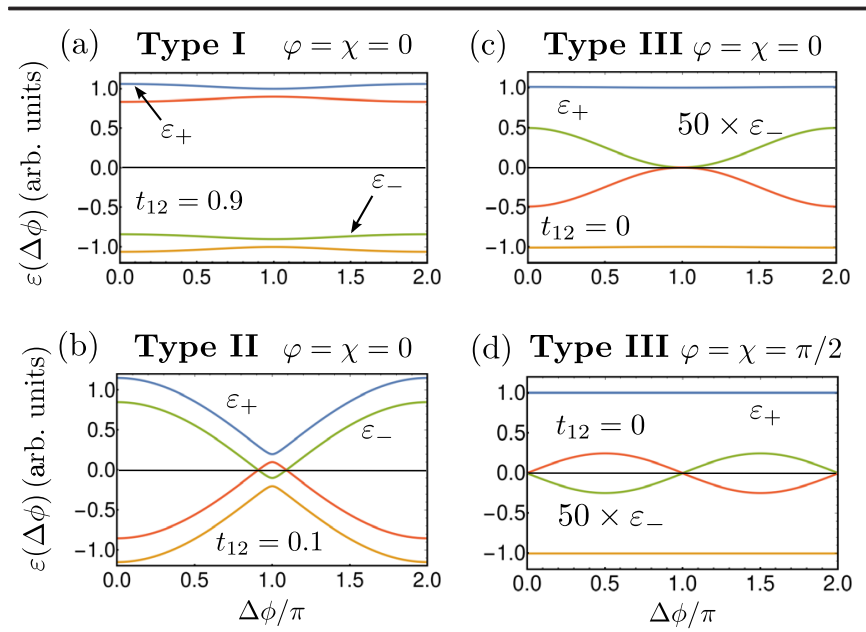

(d) Type III $\varphi=\chi=\pi / 2$

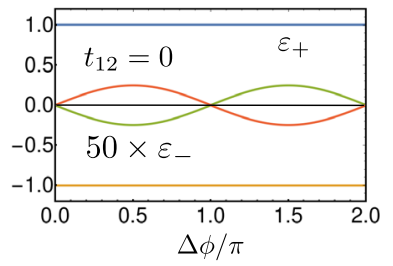

FIG. 2. ABS spectra corresponding to the three types of scenarios shown in Fig. 1, when the two TSCs are biased by a phase difference $\Delta \phi$. In case (a), we obtain a fully gapped ABS spectrum, while in case (b), the lowest ABS branch contains two FP-protected linear crossings. Case (c) can be viewed as the critical situation where the two linear crossings of case (b) merge into a single quadratic band crossing. In case (d), the $\varepsilon_{-}$branch has a sinusoidal form and contains two linear crossings at 0 and $\pi$. In (b), $t_{13}^{(0)}=t_{24}^{(0)}=1$ and $t_{34}^{(0)}=0.2$. In the rest, $t_{13}^{(0)}=t_{24}^{(0)}=0.1$ and $t_{34}^{(0)}=1$. In all panels, $t_{14,23}=0$.

of edge MBSs hybridize to ABSs, with energy dispersions $\varepsilon_{+} \approx t_{34}$ and $\varepsilon_{-} \approx-t_{12}$, for the choice $t_{34}>t_{12}>0$. Since $t_{12}$ and $t_{34}$ are independent of $\Delta \phi$, the ABS bands are very weakly dispersive with $\Delta \phi$. With the use of Eq. (2), we show such a dispersion in Fig. 2(a).

$\left|t_{12}^{(0)}\right|,\left|t_{34}^{(0)}\right| \ll\left|t_{13}^{(0)}\right|,\left|t_{24}^{(0)}\right|$. - We now consider the inverse limit, in which the symmetries protecting the multiple MBSs are weakly violated. In the fully symmetric $t_{12}=$ $t_{34}=0$ case, each isolated TSC has a symmetry-protected pair of MBSs per edge. After contact, one finds the $4 \pi$ periodic [17] ABS dispersions $\varepsilon_{+}=t_{13}$ and $\varepsilon_{-}=t_{24}$, with each one containing a single FP-protected crossing [60,62]. When $\chi=0$, the two crossings appear for the same value of $\Delta \phi$, and the symmetry-violating $t_{12}$ and $t_{34}$ terms open an energy gap at the degeneracy point. This gap opening redistributes the two initially coinciding crossings by transferring them to one of the two resulting $2 \pi$-periodic ABS dispersions. Figure 2(b) depicts such a band structure, which can be understood by separately examining the ABS dispersions near and away from the degeneracy point. Away from it, the condition $\left|t_{12}^{(0)}\right|,\left|t_{34}^{(0)}\right| \ll\left|t_{13}^{(0)}\right|,\left|t_{24}^{(0)}\right|$ implies that $\varepsilon_{+} \approx t_{13}$ and $\varepsilon_{-} \approx t_{24}$. Instead, this hierarchy becomes inverted near the degeneracy point, where the ABS energies are given by the gap-opening terms $t_{12}$ and $t_{34}$, as in Fig. 2(a). A smooth connection between the two regimes is ensured by the appearance of two linear crossings. These are FP protected and, unless a gap closing occurs, they are only removable by annihilating each other.
This is achievable, for instance, by increasing the intra-TSC MBS couplings.

$\left|t_{13}^{(0)}\right|,\left|t_{24}^{(0)}\right| \ll\left|t_{34}^{(0)}\right|$ and $\left|t_{12}^{(0)}\right|=0$. - We proceed by assuming that the two MBSs of the first TSC are uncoupled even after contacting the second TSC, which is assumed to feature a nonzero $t_{34}$, stemming from the violation of the ensuing symmetry. This violation is either externally imposed or spontaneously chosen by the hybrid system [113]. Using Eq. (2), we find $\varepsilon_{+} \approx t_{34}$ and $\varepsilon_{-} \approx t_{13} t_{24} / t_{34}$. Thus, there now exists a high- and low-energy sector for the ABSs, with only the $\varepsilon_{-}$branch exhibiting a substantial dispersion with $\Delta \phi$, since it reads:

$$
\varepsilon_{-} \propto t_{13} t_{24} \propto \cos \chi+\cos (\Delta \phi+\varphi) .
$$

For $\varphi=\chi=0$, Eq. (3) yields the quadratic crossing at $\Delta \phi=\pi$, shown in Fig. 2(c), which can be viewed as two merged linear crossings of an ABS dispersion as the one in Fig. 2(b). Another phase-offset option is $\varphi=\chi=\pi / 2$, where one obtains a $\sin (\Delta \phi)$ term, with two linear crossings at 0 and $\pi$, cf. Fig. 2(d). Additional details are given in Ref. [113] and the Supplemental Material (SM) [114]. In the former, we show that all the dispersions of Fig. 2 are accessible in a TSC junction of two spin-triplet $p$-wave SCs in the presence of Zeeman fields and spin anisotropy. As we find there, case (c) of Fig. 2 appears when the uncoupled interface MBSs are protected by either a chiral or a unitary symmetry, while case (d) arises when the two TSCs possess different chiral symmetries.

In Fig. 3 and the SM [114], we discuss alternative platforms prominent for ABS engineering. Figure 3(a) depicts two coupled single-channel semiconductor nanowires (NWs) in proximity to conventional SCs on each side of the junction. In addition, magnetic fields $\left(B_{z, 1,2,3,4}\right)$, spinorbit coupling fields $\left(\boldsymbol{\alpha}_{1,2,3,4}\right)$, and fluxes $\left(\Phi_{\ell, r}\right.$ and $\left.\Phi=\Delta \phi\right)$ are present. Here, we express the fluxes in terms of the phase differences they induce. This versatile system allows for multiple MBSs per TSC edge protected by a chiral symmetry. The latter is associated with either a Kramers degeneracy [112,120-124] $\left(\Phi_{\ell, r}=\pi\right.$ and $\left.B_{z, \ell, r}=0\right)$ or a sublattice symmetry [125-128] $\left(\Phi_{\ell, r}=0\right)$. The remaining unspecified parameters are suitably tuned within a window that allows all four NWs to be in the TSC phase simultaneously. Instead, the slightest deviations of the phases and magnetic fields from the values indicated above violate chiral symmetry and generate the couplings $t_{12,34}$. See also the SM [114].

Our results open perspectives for nontrivial topology in synthetic spaces, when the ABS spectrum is fully gapped. This becomes possible (i) in devices such as the one in Fig. 3(a) when all MBS couplings are controllable and the interface is flux or spin active, (ii) in two 1D TSC junctions of Fig. 3(a), stacked as in Fig. 3(b), with each junction supporting gapless ABS spectra, and (iii) in infinite networks of identical junctions as in Fig. 3(c). For fully gapped ABS spectra stemming from four coupled MBSs, one can 


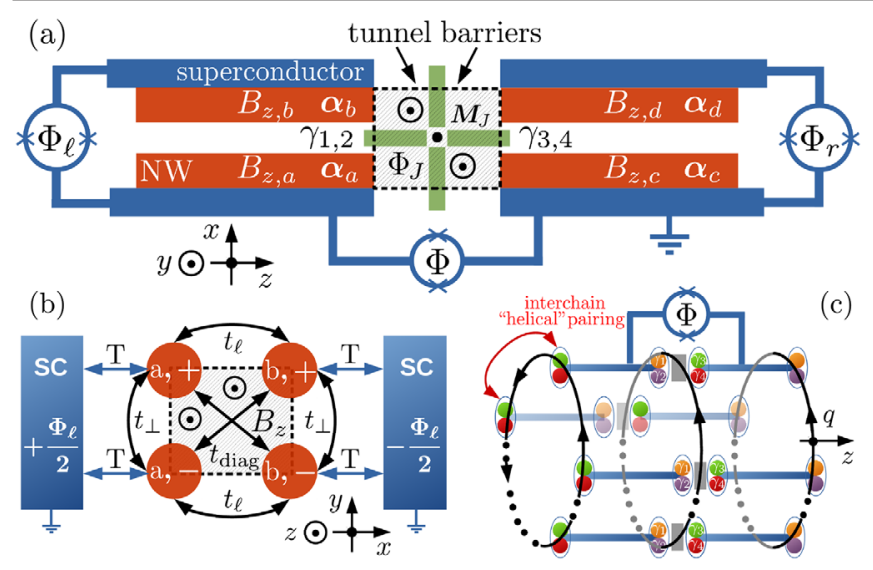

FIG. 3. (a) Junction of two TSCs, each one consisting of two coupled single-channel semiconductor nanowires (NWs) in proximity to conventional SCs. The NWs feel a spin-orbit coupling $(\boldsymbol{\alpha} \times \boldsymbol{\sigma})_{z} \hat{p}_{z}$. For specific values of fluxes $\Phi_{\ell / r}$ and magnetic fields $B_{z}$, a pair of MBSs appears on each side of the junction. Each pair is protected by a Kramers degeneracy or a sublattice symmetry, which is violated away from the sweet spot. (b) Cross section of the lhs of a device, which is obtained by stacking two junctions shown in (a). (c) "Infinite" 1D network of identical TSC junctions described by the wave number $q$. FP pumping occurs by varying $\Delta \phi=\Phi$ and an additional parameter, which in (a) is the interface flux $\Phi_{J}$ (magnetization $\boldsymbol{M}_{J}$ ), (b) is the flux $\sim B_{z}$ piercing the cross section, and in (c) is the phase of the interchain pairing [114].

decompose $i \hat{B}$, which defines a $\mathfrak{g} \mathfrak{o}(4)$ spectrum-generating algebra, into two $\mathfrak{g} \mathfrak{o}(3)$ subalgebras, i.e., $i \hat{B}=\sum_{n=1,2} \boldsymbol{g}_{n} \cdot \boldsymbol{L}_{n}$. Using the Pauli matrices $\lambda$ and $\boldsymbol{\kappa}$, the $\mathfrak{s} \mathfrak{p}(3)$ generators read $\boldsymbol{L}_{1}=\left(\lambda_{1} \kappa_{2}, \lambda_{2}, \lambda_{3} \kappa_{2}\right) / 2$ and $\boldsymbol{L}_{2}=\left(\lambda_{2} \kappa_{1}, \kappa_{2}, \lambda_{2} \kappa_{3}\right) / 2$. Therefore, $\quad g_{1}=-\left(t_{13}-t_{24}, t_{14}+t_{23}, t_{12}+t_{34}\right) \quad$ and $\boldsymbol{g}_{2}=-\left(t_{13}+t_{24}, t_{12}-t_{34}, t_{14}-t_{23}\right)$. See also Ref. [108].

$\mathbb{Z}$-FP pumping and chiral anomaly effects occur when the MBS couplings depend on an additional $2 \pi$-periodic variable $\theta$. In this case, one introduces the Berry curvature $\Omega_{\Delta \phi, \theta}^{-, n}$ [97] of the occupied eigenstates $\left|u_{-, n}(\Delta \phi, \theta)\right\rangle$ of each $\mathfrak{g} \mathfrak{o}(3)$ subalgebra with $n=1,2$. Sweeping $\Delta \phi$ and $\theta$ over an area $\mathcal{A}:\left[\Delta \phi_{i}, \Delta \phi_{f}\right] \times\left[\theta_{i}, \theta_{f}\right]$ yields the pumped charge per period $2 \pi: \Delta Q(\mathcal{A})=2 e \iint_{\mathcal{A}} d \Delta \phi d \theta \Omega_{\Delta \phi, \theta}^{-, 1} /(2 \pi)$ [114]. Here, we assumed that $\left|g_{1}\right|>\left|g_{2}\right|$, otherwise the Berry curvature $\Omega_{\Delta \phi, \theta}^{-, 2}$ should appear above [114]. Moreover, we considered a two-step adiabatic process in which the sweeping rates satisfy $\dot{\Delta \phi} \ll \dot{\theta}$, where $\dot{f} \equiv d f / d t$. For $\left|g_{1}\right| \rightarrow 0$, the $\Omega_{\Delta \phi, \theta}^{-, 1}$ peaks at the positions of the Berry monopoles, i.e., Weyl points in $(\Delta \phi, \theta, m)$ space, with $m$ an additional parameter controlling $\left|\boldsymbol{g}_{1}\right|$. In the cases of relevance, each Weyl point contributes with $\pm \frac{1}{2}$ to the integral. When $\mathcal{A} \equiv \mathbb{T}^{2}=[0,2 \pi] \times[0,2 \pi]$, we find $\Delta Q /(2 e)=C_{1} \in \mathbb{Z}$, with $C_{1}$ the respective first Chern number [97]. This implies that FP pumping is not possible, unless selective sweeping is employed, so that only an odd number of Weyl points are inside $\mathcal{A}$ for $\left|\boldsymbol{g}_{1}\right| \rightarrow 0$.
In Fig. 3(b) we find $g_{1}=(m \cos \theta, m \sin \theta, \varepsilon(\Delta \phi))$, with $\varepsilon(\Delta \phi)$ an ABS dispersion with FP-protected crossings at $\Delta \phi=\Delta \phi_{c}$, as in Figs. 2(b) and 2(d). Viewing the phase difference $\Delta \phi$ as a synthetic momentum yields a Dirac Hamiltonian $\boldsymbol{g}_{1} \cdot \boldsymbol{\kappa}$ defined in 2D $(\Delta \phi, t)$ spacetime about each crossing point since, there, $\varepsilon(\Delta \phi) \approx v_{c}\left(\Delta \phi-\Delta \phi_{c}\right)$ with $v_{c}$ the slope of the dispersion evaluated at $\Delta \phi=\Delta \phi_{c}$. Now, Berry monopoles (nodes) appear in $(\Delta \phi, m)$ space at $\left(\Delta \phi_{c}, m=0\right)$ and lead to chiral anomaly effects [98]. In the standard 2D Dirac theory, chiral anomaly manifests as the nonconservation of the chiral charge $\rho_{5} \propto\left\langle\kappa_{z}\right\rangle$ and current $j_{5} \propto\langle\mathbb{1}\rangle$ densities. Consequently, the Goldstone-Wilczek formula [129] implies that spatial and temporal variations of $\theta$ induce electric charge $\rho \propto\langle\mathbb{T}\rangle$ and current $j \propto\left\langle\kappa_{z}\right\rangle$ densities. Here, the occurrence of chiral anomaly implies

$$
\Delta Q(\mathcal{A})=-e \frac{\Delta \theta}{\pi} \sum_{c} \operatorname{sgn}\left(v_{c}\right) \int_{\mathcal{C}} d \varepsilon \delta\left(\varepsilon-\varepsilon_{c}\right),
$$

where $\Delta \theta=\theta_{f}-\theta_{i}$ and $\mathcal{C}$ denotes the ABS-dispersion path $\varepsilon_{i} \mapsto \varepsilon_{f}$, set by $\Delta \phi_{i} \mapsto \Delta \phi_{f}$. The integral is nonzero only if the path includes crossing points of $\varepsilon(\Delta \phi)$. The contribution of each crossing point is $\operatorname{sgn}\left(v_{c}\right) 1$. Thus, for ABS dispersions containing two crossings with opposite slopes, $\Delta Q$ is zero when the path includes both of them, or equivalently, a quadratic crossing. Notably, FP pumping requires $\Delta \theta=\pi$. Similar effects emerge for loosely coupled 1D junctions as in Fig. 3(c), with intrinsic or effective helical-like interchain $p$-wave pairing $\mathcal{D}(q) \sim$ $q e^{-i \theta}$ being a prerequisite [113], since $m \sim q$. The pumped charge per mode $q$ is half of that in Eq. (4) [114]; thus, FP pumping requires $\Delta \theta=2 \pi$.

In conclusion, we remark that our predictions appear experimentally feasible in organic SCs [16], purple bronze [21], and hybrid devices [84,91,92,130,131], as explained in the SM [114]. The demonstration of nonstandard ABS dispersions promises to open novel paths for the detection of chiral anomaly and FP-protected crossings, thus providing indirect evidence for MBSs. Finally, the Berrymonopole tomography proposed here is robust against disorder and parameter detunings, as long as these only modify the locations of the monopoles.

P. K. would like to thank Kasper Grove Rasmussen and Michele Burrello for helpful discussions.

*kotetes@itp.ac.cn

[1] D. Castelvecchi, Quantum computers ready to leap out of the lab in 2017, Nature (London) 541, 9 (2017).

[2] Y. Makhlin, G. Schön, and A. Shnirman, Quantum-state engineering with Josephson-junction devices, Rev. Mod. Phys. 73, 357 (2001).

[3] M. H. Devoret, A. Wallraff, and J. M. Martinis, Superconducting qubits: A short review, arXiv:cond-mat/ 0411174. 
[4] B. D. Josephson, Possible new effects in superconductive tunnelling, Phys. Lett. 1, 251 (1962).

[5] V. Ambegaokar and A. Baratoff, Tunneling Between Superconductors, Phys. Rev. Lett. 10, 486 (1963).

[6] I. Kulik and A. Omelyanchuk, Properties of superconducting microbridges in the pure limit, Sov. J. Low Temp. Phys. 3, 459 (1977).

[7] A. Furusaki and M. Tsukada, Current-carrying states in Josephson junctions, Phys. Rev. B 43, 10164 (1991).

[8] C. W. J. Beenakker and H. van Houten, Josephson Current Through a Superconducting Quantum Point Contact Shorter than the Coherence Length, Phys. Rev. Lett. 66, 3056 (1991).

[9] A. Furusaki, Josephson current carried by Andreev levels in superconducting quantum point contacts, Superlattices Microstruct. 25, 809 (1999).

[10] A. F. Andreev, The thermal conductivity of the intermediate state in superconductors, Sov. Phys. JETP 19, 1228 (1964).

[11] A. Zazunov, V. S. Shumeiko, E. N. Bratus', J. Lantz, and G. Wendin, Andreev Level Qubit, Phys. Rev. Lett. 90, 087003 (2003).

[12] M. Sigrist and K. Ueda, Phenomenological theory of unconventional superconductivity, Rev. Mod. Phys. 63, 239 (1991).

[13] Y. Tanaka, T. Hirai, K. Kusakabe, and S. Kashiwaya, Theory of the Josephson effect in a superconductor/onedimensional electron gas/superconductor junction, Phys. Rev. B 60, 6308 (1999).

[14] N. Read and D. Green, Paired states of Fermions in two dimensions with breaking of parity and time-reversal symmetries, and the fractional quantum Hall effect, Phys. Rev. B 61, 10267 (2000).

[15] D. A. Ivanov, Non-Abelian Statistics of Half-Quantum Vortices in P-Wave Superconductors, Phys. Rev. Lett. 86, 268 (2001).

[16] I. J. Lee, S. E. Brown, W. G. Clark, M. J. Strouse, M. J. Naughton, W. Kang, and P. M. Chaikin, Triplet Superconductivity in an Organic Superconductor Probed by NMR Knight Shift, Phys. Rev. Lett. 88, 017004 (2001).

[17] A. Y. Kitaev, Unpaired Majorana fermions in quantum wires, Phys. Usp. 44, 131 (2001).

[18] K. Sengupta, I.Žutić, H.-J. Kwon, V. M. Yakovenko, and S. Das Sarma, Midgap edge states and pairing symmetry of Quasi-one-dimensional organic superconductors, Phys. Rev. B 63, 144531 (2001).

[19] G. E. Volovik, The Universe in a Helium Droplet (Oxford University Press, Oxford, 2003).

[20] Y. Maeno, S. Kittaka, T. Nomura, S. Yonezawa, and K. Ishida, Evaluation of spin-triplet superconductivity in $\mathrm{Sr}_{2} \mathrm{RuO}_{4}$, J. Phys. Soc. Jpn. 81, 011009 (2012).

[21] A. G. Lebed and O. Sepper, Possible triplet superconductivity in the Quasi-one-dimensional conductor $\mathrm{Li}_{0.9} \mathrm{Mo}_{6} \mathrm{O}_{17}$, Phys. Rev. B 87, 100511 (2013).

[22] E. Dumitrescu and S. Tewari, Topological properties of the time-reversal-symmetric Kitaev chain and applications to organic superconductors, Phys. Rev. B 88, 220505(R) (2013).

[23] R. Wakatsuki, M. Ezawa, Y. Tanaka, and N. Nagaosa, Fermion fractionalization to Majorana Fermions in a dimerized Kitaev superconductor, Phys. Rev. B 90, 014505 (2014).

[24] E. Dumitrescu, J. D. Sau, and S. Tewari, Magnetic field response and Chiral symmetry of time-reversal-invariant topological superconductors, Phys. Rev. B 90, 245438 (2014).

[25] M. T. Mercaldo, M. Cuoco, and P. Kotetes, Magnetic-fieldinduced topological reorganization of a P-wave superconductor, Phys. Rev. B 94, 140503(R) (2016).

[26] M. T. Mercaldo, M. Cuoco, and P. Kotetes, Magnetic manipulation of topological states in P-wave superconductors, Physica (Amsterdam) 536B, 730 (2018).

[27] M. Sato and Y. Ando, Topological superconductors: A review, Rep. Prog. Phys. 80, 076501 (2017).

[28] L. Fu and C. L. Kane, Superconducting Proximity Effect and Majorana Fermions at the Surface of a Topological Insulator, Phys. Rev. Lett. 100, 096407 (2008).

[29] M. Z. Hasan and C. L. Kane, Colloquium: Topological insulators, Rev. Mod. Phys. 82, 3045 (2010).

[30] X.-L. Qi and S.-C. Zhang, Topological insulators and superconductors, Rev. Mod. Phys. 83, 1057 (2011).

[31] J. Alicea, New directions in the pursuit of Majorana Fermions in solid state systems, Rep. Prog. Phys. 75, 076501 (2012).

[32] C. W. J. Beenakker, Search for Majorana Fermions in superconductors, Annu. Rev. Condens. Matter Phys. 4, 113 (2013).

[33] M. Leijnse and K. Flensberg, Introduction to topological superconductivity and Majorana fermions, Semicond. Sci. Technol. 27, 124003 (2012).

[34] P. Kotetes, Classification of engineered topological superconductors, New J. Phys. 15, 105027 (2013).

[35] S. R. Elliott and M. Franz, Colloquium: Majorana fermions in nuclear, particle, and solid-state physics, Rev. Mod. Phys. 87, 137 (2015).

[36] R. Aguado, Majorana quasiparticles in condensed matter, Riv. Nuovo Cimento 40, 523 (2017).

[37] R. M. Lutchyn, E. P. A. M. Bakkers, L. P. Kouwenhoven, P. Krogstrup, C. M. Marcus, and Y. Oreg, Realizing Majorana zero modes in superconductor-semiconductor heterostructures, Nat. Rev. Mater. 3, 52 (2018).

[38] J. D. Sau, R. M. Lutchyn, S. Tewari, and S. Das Sarma, Generic New Platform for Topological Quantum Computation Using Semiconductor Heterostructures, Phys. Rev. Lett. 104, 040502 (2010).

[39] J. Alicea, Majorana Fermions in a tunable semiconductor device, Phys. Rev. B 81, 125318 (2010).

[40] R. M. Lutchyn, J. D. Sau, and S. Das Sarma, Majorana Fermions and a Topological Phase Transition in Semiconductor-Superconductor Heterostructures, Phys. Rev. Lett. 105, 077001 (2010).

[41] Y. Oreg, G. Refael, and F. von Oppen, Helical Liquids and Majorana Bound States in Quantum Wires, Phys. Rev. Lett. 105, 177002 (2010).

[42] T.-P. Choy, J. M. Edge, A. R. Akhmerov, and C. W. J. Beenakker, Majorana Fermions emerging from magnetic nanoparticles on a superconductor without spin-orbit coupling, Phys. Rev. B 84, 195442 (2011).

[43] M. Kjaergaard, K. Wölms, and K. Flensberg, Majorana Fermions in superconducting nanowires without spin-orbit coupling, Phys. Rev. B 85, 020503(R) (2012). 
[44] S. Nadj-Perge, I. K. Drozdov, B. A. Bernevig, and A. Yazdani, Proposal for realizing Majorana Fermions in chains of magnetic atoms on a superconductor, Phys. Rev. B 88, 020407(R) (2013).

[45] S. Nakosai, Y. Tanaka, and N. Nagaosa, Two-dimensional P-wave superconducting states with magnetic moments on a conventional S-wave superconductor, Phys. Rev. B 88, 180503(R) (2013).

[46] B. Braunecker and P. Simon, Interplay between Classical Magnetic Moments and Superconductivity in Quantum One-Dimensional Conductors: Toward a Self-Sustained Topological Majorana Phase, Phys. Rev. Lett. 111, 147202 (2013).

[47] J. Klinovaja, P. Stano, A. Yazdani, and D. Loss, Topological Superconductivity and Majorana Fermions in RKKY Systems, Phys. Rev. Lett. 111, 186805 (2013).

[48] M. M. Vazifeh and M. Franz, Self-Organized Topological State with Majorana Fermions, Phys. Rev. Lett. 111, 206802 (2013).

[49] F. Pientka, L. I. Glazman, and F. von Oppen, Topological superconducting phase in Helical Shiba chains, Phys. Rev. B 88, 155420 (2013).

[50] K. Pöyhönen, A. Westström, J. Röntynen, and T. Ojanen, Majorana states in Helical Shiba chains and ladders, Phys. Rev. B 89, 115109 (2014).

[51] A. Heimes, P. Kotetes, and G. Schön, Majorana Fermions from Shiba states in an antiferromagnetic chain on top of a superconductor, Phys. Rev. B 90, 060507(R) (2014).

[52] P. M. R. Brydon, S. Das Sarma, H.-Y. Hui, and J. D. Sau, Topological Yu-Shiba-Rusinov chain from spin-orbit coupling, Phys. Rev. B 91, 064505 (2015).

[53] J. Li, H. Chen, I. K. Drozdov, A. Yazdani, B. A. Bernevig, and A.H. MacDonald, Topological superconductivity induced by ferromagnetic metal chains, Phys. Rev. B 90, 235433 (2014).

[54] A. Heimes, D. Mendler, and P. Kotetes, Interplay of topological phases in magnetic Adatom-chains on top of a Rashba superconducting surface, New J. Phys. 17, 023051 (2015).

[55] J. Xiao and J. An, Chiral symmetries and Majorana Fermions in coupled magnetic atomic chains on a superconductor, New J. Phys. 17, 113034 (2015).

[56] M. Schecter, K. Flensberg, M. H. Christensen, B. M. Andersen, and J. Paaske, Self-organized topological superconductivity in a Yu-Shiba-Rusinov chain, Phys. Rev. B 93, 140503(R) (2016).

[57] S. Hoffman, J. Klinovaja, and D. Loss, Topological phases of inhomogeneous superconductivity, Phys. Rev. B 93, 165418 (2016).

[58] G. M. Andolina and P. Simon, Topological properties of chains of magnetic impurities on a superconducting substrate: Interplay between the Shiba band and ferromagnetic wire limits, Phys. Rev. B 96, 235411 (2017).

[59] H.-J. Kwon, K. Sengupta, and V. M. Yakovenko, Fractional AC Josephson effect in unconventional superconductors, Low Temp. Phys. 30, 613 (2004).

[60] L. Fu and C. L. Kane, Josephson current and noise at a superconductor-quantum spin Hall insulator-superconductor junction, Phys. Rev. B 79, 161408(R) (2009).
[61] Y. Tanaka, T. Yokoyama, and N. Nagaosa, Manipulation of the Majorana Fermion, Andreev Reflection, and Josephson Current on Topological Insulators, Phys. Rev. Lett. 103, 107002 (2009).

[62] C. W. J. Beenakker, D. I. Pikulin, T. Hyart, H. Schomerus, and J. P. Dahlhaus, Fermion-Parity Anomaly of the Critical Supercurrent in the Quantum Spin-Hall Effect, Phys. Rev. Lett. 110, 017003 (2013).

[63] P. Kotetes, A. Shnirman, and G. Schön, Engineering and manipulating topological qubits in 1D quantum wires, J. Korean Phys. Soc. 62, 1558 (2013).

[64] L. Jiang, D. Pekker, J. Alicea, G. Refael, Y. Oreg, A. Brataas, and F. von Oppen, Magneto-Josephson effects in junctions with Majorana bound states, Phys. Rev. B 87, 075438 (2013).

[65] D. Sticlet, C. Bena, and P. Simon, Josephson effect in superconducting wires supporting multiple Majorana edge states, Phys. Rev. B 87, 104509 (2013).

[66] F. Pientka, L. Jiang, D. Pekker, J. Alicea, G. Refael, Y. Oreg, and F. von Oppen, Magneto-Josephson effects and Majorana bound states in quantum wires, New J. Phys. 15, 115001 (2013).

[67] J. Cayao, A. M. Black-Schaffer, E. Prada, and R. Aguado, Andreev spectrum and supercurrents in nanowire-based SNS junctions containing Majorana bound states, Beilstein J. Nanotechnol. 9, 1339 (2018).

[68] Y. Tanaka and S. Kashiwaya, Local density of states of quasiparticles near the interface of nonuniform d-wave superconductors, Phys. Rev. B 53, 9371 (1996).

[69] S. Kashiwaya and Y. Tanaka, Tunnelling effects on surface bound states in unconventional superconductors, Rep. Prog. Phys. 63, 1641 (2000).

[70] A. Y. Kitaev, Fault-tolerant quantum computation by anyons, Ann. Phys. (Amsterdam) 303, 2 (2003).

[71] C. Nayak, S. H. Simon, A. Stern, M. Freedman, and S. Das Sarma, Non-Abelian anyons and topological quantum computation, Rev. Mod. Phys. 80, 1083 (2008).

[72] J. Alicea, Y. Oreg, G. Refael, F. von Oppen, and M. P. A. Fisher, Non-Abelian statistics and topological quantum information processing in 1D wire networks, Nat. Phys. 7, 412 (2011).

[73] Jian Li, T. Neupert, B. A. Bernevig, and A. Yazdani, Manipulating Majorana zero modes on atomic rings with an external magnetic field, Nat. Commun. 7, 10395 (2016).

[74] V. Mourik, K. Zuo, S. M. Frolov, S. R. Plissard, E. P. A. M. Bakkers, and L. P. Kouwenhoven, Signatures of Majorana Fermions in hybrid superconductor-semiconductor nanowire devices, Science 336, 1003 (2012).

[75] S. Nadj-Perge, I. K. Drozdov, Jian Li, Hua Chen, S. Jeon, J. Seo, A. H. MacDonald, B. A. Bernevig, and A. Yazdani, Observation of Majorana Fermions in ferromagnetic atomic chains on a superconductor, Science 346, 602 (2014).

[76] S. Hart, H. Ren, T. Wagner, P. Leubner, M. Mühlbauer, C. Brüne, H. Buhmann, L W. Molenkamp, and A. Yacoby, Induced superconductivity in the quantum spin Hall edge, Nat. Phys. 10, 638 (2014).

[77] S.-P. Lee, K. Michaeli, J. Alicea, and A. Yacoby, Revealing Topological Superconductivity in Extended Quantum Spin 
Hall Josephson Junctions, Phys. Rev. Lett. 113, 197001 (2014).

[78] M. Ruby, F. Pientka, Y. Peng, F. von Oppen, B. W. Heinrich, and K. J. Franke, End States and Subgap Structure in Proximity-Coupled Chains of Magnetic Adatoms, Phys. Rev. Lett. 115, 197204 (2015).

[79] R. Pawlak, M. Kisiel, J. Klinovaja, T. Meier, S. Kawai, T. Glatzel, D. Loss, and E. Meyer, Probing atomic structure and Majorana wavefunctions in mono-atomic Fe-chains on superconducting Pb-surface, npj Quantum Inf. 2, 16035 (2016).

[80] H.-H. Sun, K.-W. Zhang, L.-H. Hu, C. Li, G.-Y. Wang, H.-Y. Ma, Z.-A. Xu, C.-L. Gao, D.-D. Guan, Y.-Y. Li, C. Liu, D. Qian, Yi Zhou, L. Fu, S.-C. Li, F.-C. Zhang, and J.-F. Jia, Observation of Majorana Fermions with Spin Selective Andreev Reflection in the Vortex of Topological Superconductor, Phys. Rev. Lett. 116, 257003 (2016).

[81] J. Wiedenmann, E. Bocquillon, R. S. Deacon, S. Hartinger, O. Herrmann, T. M. Klapwijk, L. Maier, C. Ames, C. Brüne, C. Gould, A. Oiwa, K. Ishibashi, S. Tarucha, H. Buhmann, and L. W. Molenkamp, $4 \pi$-periodic Josephson supercurrent in HgTe-based topological Josephson junctions, Nat. Commun. 7, 10303 (2016).

[82] M. T. Deng, S. Vaitikiènas, E. B. Hansen, J. Danon, M. Leijnse, K. Flensberg, P. Krogstrup, and C. M. Marcus, Majorana bound state in a coupled quantum-dot hybridnanowire system, Science 354, 1557 (2016).

[83] S. M. Albrecht, A. P. Higginbotham, M. Madsen, F. Kuemmeth, T. S. Jespersen, J. Nygård, P. Krogstrup, and C. M. Marcus, Exponential protection of zero modes in Majorana Islands, Nature (London) 531, 206 (2016).

[84] F. Nichele, A. C. C. Drachmann, A. M. Whiticar, E. C. T. O'Farrell, H. J. Suominen, A. Fornieri, T. Wang, G. C. Gardner, C. Thomas, A. T. Hatke, P. Krogstrup, M. J. Manfra, K. Flensberg, and C. M. Marcus, Scaling of Majorana Zero-Bias Conductance Peaks, Phys. Rev. Lett. 119, 136803 (2017).

[85] Q. L. He, L. Pan, A. L. Stern, E. C. Burks, X. Che, G. Yin, J. Wang, B. Lian, Q. Zhou, E. S. Choi, K. Murata, X. Kou, Z. Chen, T. Nie, Q. Shao, Y. Fan, S.-C. Zhang, K. Liu, J. Xia, and K. L. Wang, Chiral Majorana Fermion modes in a quantum anomalous Hall insulator-superconductor structure, Science 357, 294 (2017).

[86] S. Jeon, Y. Xie, J. Li, Z. Wang, B. A. Bernevig, and A. Yazdani, Distinguishing a Majorana zero mode using spinresolved measurements, Science 358, 772 (2017).

[87] L. Bours, B. Sothmann, M. Carrega, E. Strambini, E. M. Hankiewicz, L. W. Molenkamp, and F. Giazotto, A topological SQUIPT based on Helical edge states in proximity to superconductors, Phys. Rev. Applied 10, 014027 (2018).

[88] H. Zhang, C.-X. Liu, S. Gazibegovic, D. Xu, J. A. Logan, G. Wang, N. van Loo, J. D. S. Bommer, M. W. A. de Moor, D. Car, R. L. M. Op het Veld, P. J. van Veldhoven, S. Koelling, M. A. Verheijen, M. Pendharkar, D. J. Pennachio, B. Shojaei, J. S. Lee, C. J. Palmstrom, E. P. A. M. Bakkers, S. D. Sarma, and L. P. Kouwenhoven, Quantized Majorana conductance, Nature (London) 556, 74 (2018).

[89] D. Laroche, D. Bouman, D. J. van Woerkom, A. Proutski, C. Murthy, D. I. Pikulin, C. Nayak, R. J. J. van Gulik,
J. Nygård, P. Krogstrup, L. P. Kouwenhoven, and A. Geresdi, Observation of the $4 \pi$-periodic Josephson effect in InAs nanowires, Nat. Commun. 10, 245 (2019).

[90] H. Kim, A. Palacio-Morales, T. Posske, L. Rózsa, K. Palotás, L. Szunyogh, M. Thorwart, and R. Wiesendanger, Toward tailoring Majorana bound states in artificially constructed magnetic atom chains on elemental superconductors, Sci. Adv. 4, eaar5251 (2018).

[91] A. Fornieri, A. M. Whiticar, F. Setiawan, E. Portolés Marín, A. C. C. Drachmann, A. Keselman, S. Gronin, C. Thomas, T. Wang, R. Kallaher, G. C. Gardner, E. Berg, M. J. Manfra, A. Stern, C. M. Marcus, and F. Nichele, Evidence of topological superconductivity in planar Josephson junctions, Nature (London) 569, 89 (2019).

[92] H. Ren, F. Pientka, S. Hart, A. Pierce, M. Kosowsky, L. Lunczer, R. Schlereth, B. Scharf, E. M. Hankiewicz, L. W. Molenkamp, B. I. Halperin, and A. Yacoby, Topological superconductivity in a phase-controlled Josephson junction, Nature (London) 569, 93 (2019).

[93] D. Aasen, M. Hell, R. V. Mishmash, A. Higginbotham, J. Danon, M. Leijnse, T. S. Jespersen, J. A. Folk, C. M. Marcus, K. Flensberg, and J. Alicea, Milestones toward Majorana-Based Quantum Computing, Phys. Rev. X 6, 031016 (2016).

[94] T. Karzig, C. Knapp, R. M. Lutchyn, P. Bonderson, M. B. Hastings, C. Nayak, J. Alicea, K. Flensberg, S. Plugge, Y. Oreg, C. M. Marcus, and M. H. Freedman, Scalable designs for quasiparticle-poisoning-protected topological quantum computation with Majorana zero modes, Phys. Rev. B 95, 235305 (2017).

[95] Tommy Li, W. A. Coish, M. Hell, K. Flensberg, and M. Leijnse, Four-Majorana qubit with charge readout: Dynamics and decoherence, Phys. Rev. B 98, 205403 (2018).

[96] M. V. Berry, Quantal phase factors accompanying adiabatic changes, Proc. R. Soc. A 392, 45 (1984).

[97] D. Xiao, M.-C. Chang, and Q. Niu, Berry phase effects on electronic properties, Rev. Mod. Phys. 82, 1959 (2010).

[98] S. Weinberg, The Quantum Theory of Fields (Cambridge University Press, New York, 1996).

[99] A. Altland and M. R. Zirnbauer, Nonstandard symmetry classes in mesoscopic normal-superconducting hybrid structures, Phys. Rev. B 55, 1142 (1997).

[100] A. Kitaev, Periodic table for topological insulators and superconductors, AIP Conf. Proc. 1134, 22 (2009).

[101] S. Ryu, A. Schnyder, A. Furusaki, and A. Ludwig, Topological insulators and superconductors: Ten-fold way and dimensional hierarchy, New J. Phys. 12, 065010 (2010).

[102] J. C. Y. Teo and C. L. Kane, Topological defects and gapless modes in insulators and superconductors, Phys. Rev. B 82, 115120 (2010).

[103] K. Shiozaki and M. Sato, Topology of crystalline insulators and superconductors, Phys. Rev. B 90, 165114 (2014).

[104] C.-K. Chiu, J. C. Y. Teo, A. P. Schnyder, and S. Ryu, Classification of topological quantum matter with symmetries, Rev. Mod. Phys. 88, 035005 (2016).

[105] A. Celi, P. Massignan, J. Ruseckas, N. Goldman, I. B. Spielman, G. Juzeliūnas, and M. Lewenstein, Synthetic Gauge Fields in Synthetic Dimensions, Phys. Rev. Lett. 112, 043001 (2014). 
[106] R.-P. Riwar, M. Houzet, J. S. Meyer, and Y. V. Nazarov, Multi-terminal Josephson junctions as topological matter, Nat. Commun. 7, 11167 (2016).

[107] H.-Y. Xie, M. G. Vavilov, and A. Levchenko, Weyl nodes in Andreev spectra of multiterminal Josephson junctions: Chern numbers, conductances, and supercurrents, Phys. Rev. B 97, 035443 (2018).

[108] M. Houzet and J. S. Meyer, Majorana-Weyl crossings in topological multiterminal junctions, Phys. Rev. B 100, 014521 (2019).

[109] L. J. Geerligs, S. M. Verbrugh, P. Hadley, J. E. Mooij, H. Pothier, P. Lafarge, C. Urbina, D. Esteve, and M. H. Devoret, Single Cooper pair pump, Z. Phys. B 85, 349 (1991).

[110] J. P. Pekola, J. J. Toppari, M. Aunola, M. T. Savolainen, and D. V. Averin, Adiabatic transport of Cooper pairs in arrays of Josephson junctions, Phys. Rev. B 60, R9931 (1999).

[111] D. J. Thouless, Quantization of particle transport, Phys. Rev. B 27, 6083 (1983).

[112] A. Keselman, L. Fu, A. Stern, and E. Berg, Inducing TimeReversal-Invariant Topological Superconductivity and Fermion Parity Pumping in Quantum Wires, Phys. Rev. Lett. 111, 116402 (2013).

[113] M. T. Mercaldo, P. Kotetes, and M. Cuoco, companion paper, Magnetoelectrically tunable Andreev bound state spectra and spin polarization in $p$-wave Josephson junctions, Phys. Rev. B 100, 104519 (2019).

[114] See Supplemental Material at http://link.aps.org/ supplemental/10.1103/PhysRevLett.123.126802 for additional technical details regarding our theoretical analysis, as well as an in-depth presentation of the several experimental platforms discussed in the Letter. Specifically, the various sections provide further information about (I) the Andreev-bound-state band structures in Fig. 2, (II) the Hamiltonian describing each one of the two artificial topological superconductors appearing in Fig. 3(a) and their topological properties, (III) the Majorana-bound-state couplings for a junction as in Fig. 3(a), (IV) the hybrid device of Fig. 3(b), (V) the 1D network of topological superconductor junctions depicted in Fig. 3(c), (VI) the general theory for the here-proposed fermion-parity pumping scheme, and (VII) details and guidance for the experimental realization of fermion-parity pumping in all the devices discussed in the main text. It includes Refs. [34,40,41,72,97,99-101,112,113,115-119].

[115] A. E. G. Mikkelsen, P. Kotetes, P. Krogstrup, and K. Flensberg, Hybridization at Superconductor-Semiconductor Interfaces, Phys. Rev. X 8, 031040 (2018).

[116] A. E. Antipov, A. Bargerbos, G. W. Winkler, B. Bauer, E. Rossi, and R. M. Lutchyn, Effects of Gate-Induced Electric
Fields on Semiconductor Majorana Nanowires, Phys. Rev. X 8, 031041 (2018).

[117] R. Winkler, Spin-Orbit Coupling Effects in Two-Dimensional Electron and Hole Systems (Springer, Berlin, 2003).

[118] A. C. Potter and P. A. Lee, Engineering a $p+i p$ superconductor: Comparison of topological insulator and Rashba spin-orbit coupled materials, Phys. Rev. B 83, 184520 (2011).

[119] R. Peierls, On the theory of diamagnetism from conduction electrons, Z. Phys. 80, 763 (1933).

[120] E. Gaidamauskas, J. Paaske, and K. Flensberg, Majorana Bound States in Two-Channel Time-Reversal-Symmetric Nanowire Systems, Phys. Rev. Lett. 112, 126402 (2014).

[121] A. Haim, A. Keselman, E. Berg, and Y. Oreg, Timereversal-invariant topological superconductivity induced by repulsive interactions in quantum wires, Phys. Rev. B 89, 220504(R) (2014).

[122] J. Klinovaja and D. Loss, Time-reversal invariant parafermions in interacting Rashba nanowires, Phys. Rev. B 90, 045118 (2014).

[123] C. Schrade, A. A. Zyuzin, J. Klinovaja, and D. Loss, Proximity-Induced Josephson $\pi$-Junctions in Topological Insulators, Phys. Rev. Lett. 115, 237001 (2015).

[124] A. Haim and Y. Oreg, Time-reversal-invariant topological superconductivity, arXiv:1809.06863.

[125] S. Tewari and J. D. Sau, Topological Invariants for SpinOrbit Coupled Superconductor Nanowires, Phys. Rev. Lett. 109, 150408 (2012).

[126] P. Kotetes, Topological superconductivity in Rashba semiconductors without a Zeeman field, Phys. Rev. B 92, 014514 (2015).

[127] M. Hell, M. Leijnse, and K. Flensberg, Two-Dimensional Platform for Networks of Majorana Bound States, Phys. Rev. Lett. 118, 107701 (2017).

[128] F. Pientka, A. Keselman, E. Berg, A. Yacoby, A. Stern, and B. I. Halperin, Topological Superconductivity in a Planar Josephson Junction, Phys. Rev. X 7, 021032 (2017).

[129] J. Goldstone and F. Wilczek, Fractional Quantum Numbers on Solitons, Phys. Rev. Lett. 47, 986 (1981).

[130] S. R. Plissard, I. van Weperen, D. Car, M. A. Verheijen, G. W. G. Immink, J. Kammhuber, L. J. Cornelissen, D. B. Szombati, A. Geresdi, S. M. Frolov, L. P. Kouwenhoven, and E. P. A. M. Bakkers, Formation and electronic properties of InSb nanocrosses, Nat. Nanotechnol. 8, 859 (2013).

[131] F. Krizek, J. E. Sestoft, P. Aseev, S. Marti-Sanchez, S. Vaitiekènas, L. Casparis, S. A. Khan, Y. Liu, T. Stankevič, A. M. Whiticar, A. Fursina, F. Boekhout, R. Koops, E. Uccelli, L. P. Kouwenhoven, C. M. Marcus, J. Arbiol, and P. Krogstrup, Field effect enhancement in buffered quantum nanowire networks, Phys. Rev. Mater. 2, 093401 (2018). 